\title{
A SCANNING SCHEIMPFLUG LIDAR SYSTEM DEVELOPED FOR URBAN POLLUTION MONITORING Yang Yang ${ }^{1}$, Peng Guan ${ }^{1}, \&$ Liang Mei ${ }^{1 *}$ \\ ${ }^{1}$ School of Optoelectronic Engineering and Instrumentation Science, Dalian University of Technology, Dalian 116024, China,.*meiliang@dlut.edu.cn
}

\begin{abstract}
A scanning Scheimpflug lidar system based on the Scheimpflug principle has been developed by employing a high power multimode $808 \mathrm{~nm}$ laser diode and a highly integrated CMOS sensor in Dalian University of Technology, Dalian, Northern China. Atmospheric scanning measurements in urban area were performed for the studies of particle emission sources.
\end{abstract}

\section{INTRODUCTION}

Air pollution, especially particulate pollution, has been a serious environmental problem as the rapid development of industrialization and urbanization during past decades in China. By transmitting a nanosecond-scale pulsed laser (e.g., Nd:YAG) beam into atmosphere and capturing the backscattered light from particles/molecules via e.g., photomultiplier tubes (PMT), conventional pulsed lidar techniques have been widely employed for atmospheric particle and gas monitoring since 1960s [1-5]. As a remote sensing technique, lidar is capable of real-time monitoring of atmospheric particle distribution and variation for a large area, e.g., urban area pollution monitoring, while local point monitoring instruments are only able to measure localized pollutants. Extensive work has been pursued by utilizing scanning atmospheric lidar systems for remote monitoring of atmospheric pollutants and/or emission sources. For instance, Scott M. Spuler et. al., presented a field-deployable scanning lidar system with a blind range of about $500 \mathrm{~m}$ based on an eye-safe laser $(1.54 \mu \mathrm{m})$ [6]. T. Y. He, et. al., presented a scanning lidar system employing a pulsed Nd:YAG laser, which was capable of tracking two-dimensional spatial and temporal particle distribution with an angular resolution of $0.1^{\circ}$ [7]. However, the near-range particle distribution could not be resolved due to the long blind range, i.e., $0.8 \mathrm{~km}$ of the lidar system.
The lidar systems discussed above mainly employ high-cost and sophisticated laser sources, e.g., Nd: YAG lasers and require a data acquisition unit with high sampling rate and large dynamic range to achieve high spatial resolution and long detection range. Furthermore, a common issue of the pulsed lidar systems mentioned above is of long blind range because of incomplete geometric overlap between the transmitter and receiver. Recently, a new lidar technique, referred to as the Scheimpflug lidar has been demonstrated for atmospheric remote sensing [8-13]. The Scheimpflug lidar technique is based on the Scheimpflug principle, which implies that the backscattered light of the entire aerosol volume illuminated by the laser beam can be detected by a tilted image sensor if the image sensor plane, the lens plane and the laser beam plane intersect with each other. The pixels of the CCD or CMOS sensors correspond to the distances of the illuminated volume. The Scheimpflug lidar technique can achieve a short blind range less than $100 \mathrm{~m}$ or even up to $30 \mathrm{~m}$ by employing a long senor (e.g., $28 \mathrm{~mm}$ length).

In this work, we developed a scanning Scheimpflug lidar system which was capable of scanning in both azimuth and elevation angle with $1^{\circ}$ angular resolution and short blind range. Field campaign was performed by monitoring particle distribution in Dalian, Northern China during a haze weather condition. The performance and feasibility of employing the scanning Scheimpflug lidar system for tracking emission sources is investigated.

\section{METHODOLOGY}

The schematic of the scanning Scheimpflug lidar system is shown in Figure 1. A C-mount 808-nm laser diode with $4 \mathrm{~W}$ output power is employed as the light source. The laser diode chip is a widestripe with an emission area of $230 \mu \mathrm{m}$ (slow axis) 
$\times 1 \mu \mathrm{m}$ (fast axis). As has been discussed in [9], the fast axis should be oriented along the plane of the optical layout in order to optimize the effective spatial resolution. To suppress the background noise, an 808-nm interference filter with $3 \mathrm{~nm}$ full width at half maximum (FWHM) and a high-pass color filter (RG780) with $780 \mathrm{~nm}$ cut-off wavelength are employed. Besides, multimode laser diodes typically have large uncertainties (e.g., $\pm 3 \mathrm{~nm}$ ) in terms of center emission wavelength. To optimize the transmission through the interference filter and stabilize the system performance, the 808 -nm laser diode is housed by a home-made mount with large-capacity thermoelectric cooler to control the case temperature of the laser diode.

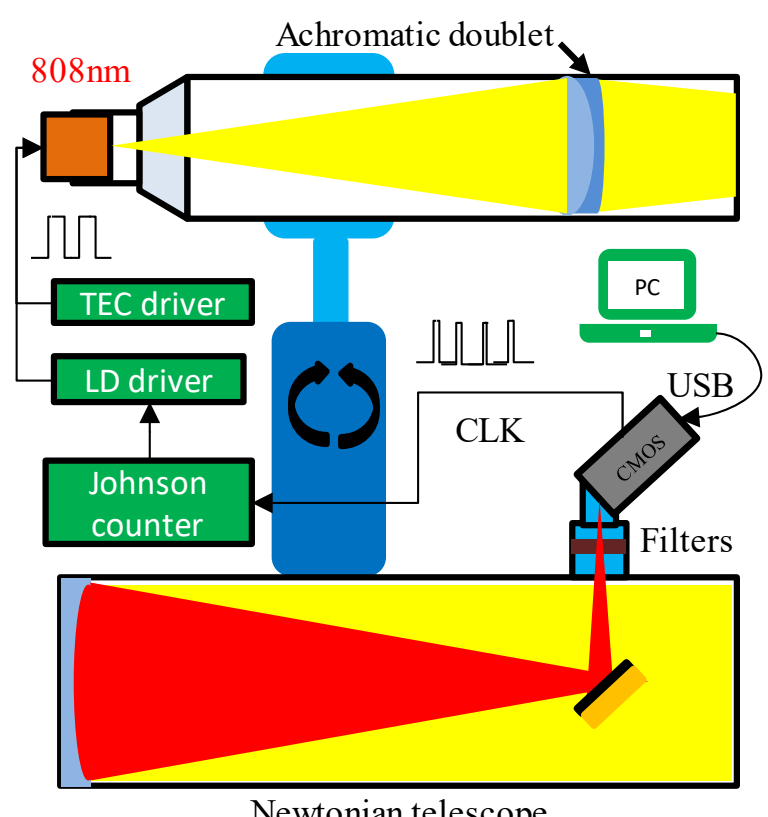

Newtonian telescope

Figure 1 Scanning Scheimpflug lidar system.

A Newtonian telescope (Skywatcher, focal length $800 \mathrm{~mm}, \varnothing 200 \mathrm{~mm}$ ) with approximately 806 $\mathrm{mm}$ separation to the laser beam collimator is employed to collect the backscattering light of the laser beam. A 2D camera (CMOSIS CMV2000, $2048 \times 1088,5.5 \mu \mathrm{m}$ ), is employed to image the profile of the laser beam. A trigger signal is generated by the camera and fed to a Johnson counter that is employed as a two-frequency divider. Thus, the laser diode is turned on and off, while synchronizing with the exposure of the camera. The camera can then record the images of the laser beam and the background alternatively.
The background as well as the dark current of the camera can thus be subtracted from the recorded raw images. The whole setup is assembled on an equatorial mount (Skywatcher, EQ6) which is capable of scanning the atmosphere in vertical and horizontal directions. A LabVIEW-based program was developed by our group to alternatively scan the lidar system and perform the measurements, according to user-set configurations.

\section{MEASUREMENTS AND RESULTS}

Field campaign was performed to scan the atmosphere of the south area of Ganjingzi District in Dalian on November $4^{\text {th }} 2016$. The scanning Scheimpflug lidar system was placed on a roof of a tall building (7 floors) in order to obtain a wide field of view. The elevation angle of the system was set to $3^{\circ}$ to avoid some tall buildings within the field of view. The scanning range covers $90^{\circ}$ with angular resolution of $1^{\circ}$, and the system was programed to work periodically and continuously. Before the measurement, the Scheimpflug lidar system is calibrated first in order to obtain the precise relationship between the position of pixel and the measuring distance, as discussed before. Figure 2 shows the scanning region and the location of the Scheimpflug lidar system and the calibration target.

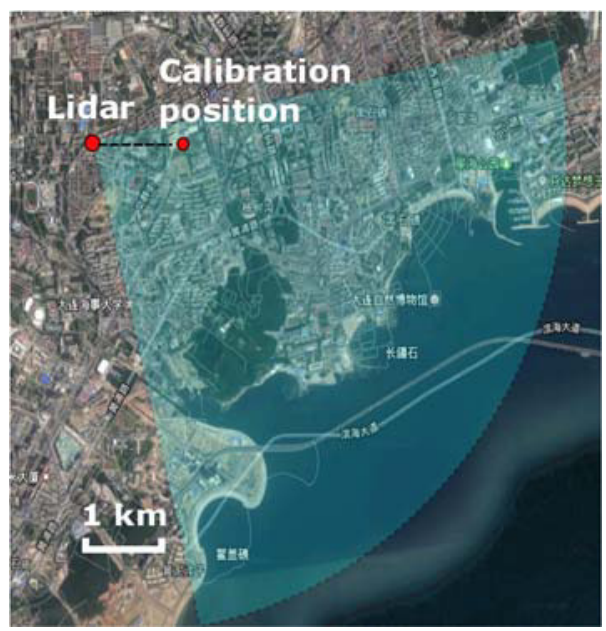

Figure 2. Top view of the scanning region (the blue sector) and the Scheimpflug lidar site.

Scanning lidar signals were recorded over a few hours. Linear interpolation and median analysis were employed to process the recorded lidar curves, in order to eliminate the influence of the swift variation of atmosphere and extremely 
strong sunlight background, as discussed in previous work. Since atmospheric aerosol distribution is changing all the time, high scanning rate is necessary to capture the spatial and temporal particle distributions. However, high scanning rate often implies a short measurement time (less signal-averaging times) and thus lower signal-tonoise ratio (SNR). To improve the SNR and thus achieve a relatively higher scanning rate, a lowpass finite response (FIR) filter with a Hanning window function was employed to suppress the solar radiation (shot) noise, readout noise and dark current noise. As shown in Figure 3, the SNR ratio was improved by a factor of 6 after employing the low-pass filter. The scanning speed of the Scheimpflug lidar system is $12 \mathrm{~min} / 90^{\circ}$ with an angular resolution of $1^{\circ}$, allowing fast monitoring of the urban particle distributions.

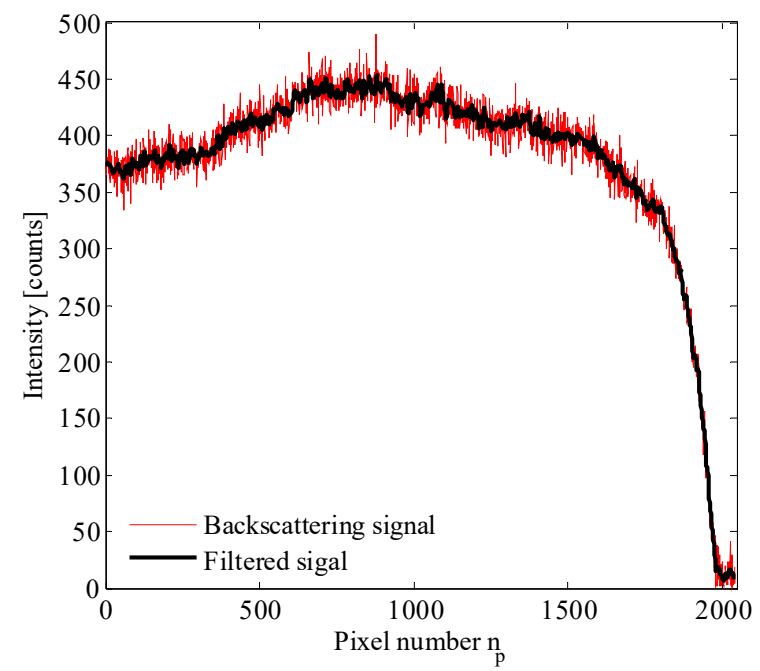

Figure 3. Backscattering signal (50 times averaging, approximately 2 seconds) recorded by the image sensor at a certain angle and the signal after applying a low-pass filter.

Figure 4 shows 2D maps of atmospheric backscattering intensities measured by the scanning Scheimpflug lidar system. As can be seen, two emission sources with substantially stronger atmospheric backscattering signal were identified, which were figured out to be two heating plants. From the backscattering signal evolution in 7 hours, it can be concluded that the maximum emission of the two plants occurred at around 16:00. Heating plants are actually one of the main sources of atmospheric pollution and causing haze weather in northern China during winter.
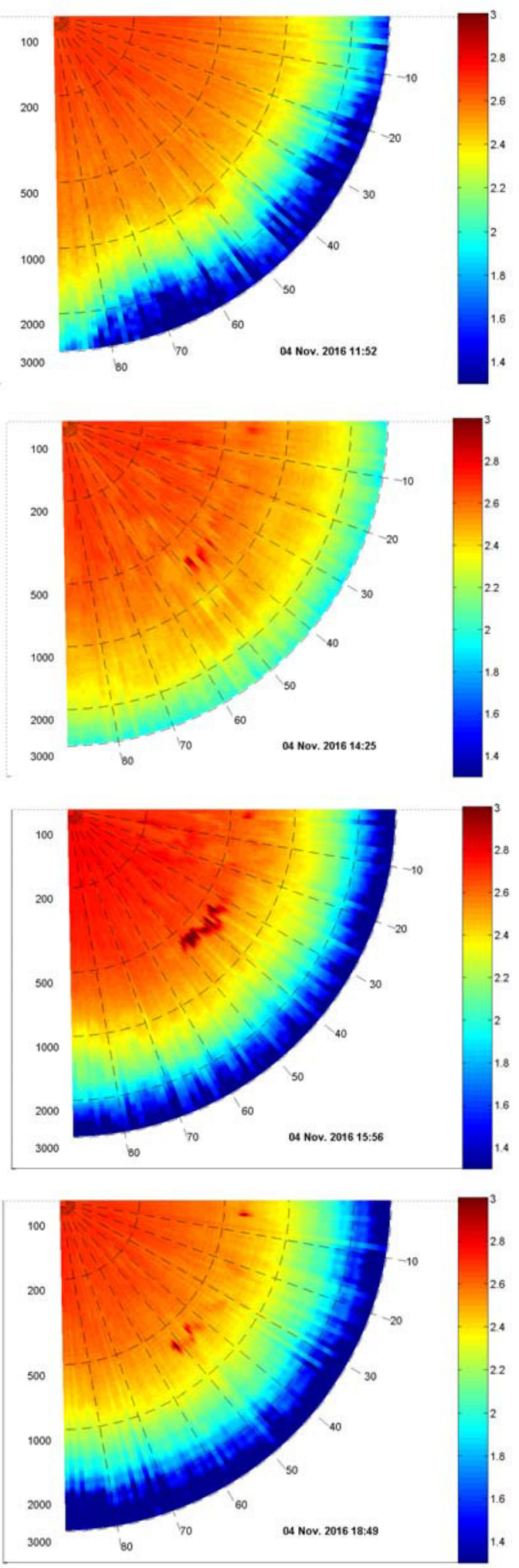

Figure 4. 2D maps of the backscattering intensities measured by the scanning lidar system. The intensities are plotted in log-scale, and y-axis is distance in meter. 


\section{CONCLUSIONS}

In urban areas, aerosol sources could be difficult to localize by single point ground-based aerosol monitoring stations due to the spatial and temporal dispersions of particles. Since the aerosol has been increasingly severe in big cities, aerosol sources identification is highly necessary for pollution control and managements. The scanning Scheimpflug lidar system developed in this work is capable of tracking the locations of the emission sources in urban area, and it is of low cost by employing continuous-wave laser diodes and image sensors.

A field campaign was performed to monitor urban particle emissions in Ganjingzi District, Dalian on November $4^{\text {th }} 2016$. The scanning speed of the scanning Scheimpflug lidar system is 12 $\min / 90^{\circ}$ with an angular resolution of $1^{\circ}$. During the campaign, two pollutant sources are identified clearly from the two-dimension figures. The blind range of the scanning Scheimpflug lidar system is very short, i.e., $80 \mathrm{~m}$ and thus near-range particles can be detected as well. During the campaign, we found out that the most time-consuming procedure for the measurement is actually the movement of the equatorial EQ6. To achieve faster scanning and higher time and spatial resolution, the scanning mechanics should be furthered improved. Field campaign with improved performance is planned in the near future.

\section{ACKNOWLEDGEMENTS}

This work was supported by the National key research and development program of China (2016YFC0200600); Fundamental Research Funds for the Central Universities (DUT15RC(3)107); Natural Science Foundation of Liaoning Province, China (201602163).

\section{References}

[1] A. Papayannis, D. Balis, V. Amiridis, G. Chourdakis, G. Tsaknakis, C. Zerefos, A. D. A. Castanho, S. Nickovic, S. Kazadzis, and J. Grabowski, 2005: Measurements of Saharan dust aerosols over the Eastern Mediterranean using elastic backscatter-Raman lidar, spectrophotometric and satellite observations in the frame of the EARLINET project, Atmos. Chem. Phys. 5, 20652079.
[2] Z. G. Guan, P. Lundin, L. Mei, G. Somesfalean, and S. Svanberg, 2010: Vertical lidar sounding of atomic mercury and nitric oxide in a major Chinese city, Appl. Phys. B 101, 465-470.

[3] J. Lenoble, L. Remer, and D. Tanre, Aerosol Remote Sensing. (Springer, Verlag Berlin Heidelberg, 2013).

[4] L. Mei, G. Y. Zhao, and S. Svanberg, 2014: Differential absorption lidar system employed for background atomic mercury vertical profiling in south China, Opt. Laser Eng. 55, 128-135.

[5] M. R. Perrone, F. De Tomasi, and G. P. Gobbi, 2014: Vertically resolved aerosol properties by multiwavelength lidar measurements, Atmos. Chem. Phys. 14, 1185-1204.

[6] S. M. Spuler and S. D. Mayor, 2004: Scanning EyeSafe Elastic Backscatter Lidar at $1.54 \mu \mathrm{m}$, Journal of Atmospheric \& Oceanic Technology 22, 696.

[7] T. Y. He, S. Stanic, F. Gao, K. Bergant, D. Veberic, X. Q. Song, and A. Dolzan, 2012: Tracking of urban aerosols using combined LIDAR-based remote sensing and ground-based measurements, Atmos. Meas. Tech. 5, 891-900.

[8] M. Brydegaard, A. Gebru, and S. Svanberg, 2014: Super resolution laser radar with blinking atmospheric particles - Application to interacting flying insects, PIER 147, 141-151.

[9] L. Mei and M. Brydegaard, 2015: Atmospheric aerosol monitoring by an elastic Scheimpflug lidar system, Opt. Exp. 23, 247841.

[10] L. Mei and M. Brydegaard, 2015: Continuous-wave differential absorption lidar, Laser \& Photon. Rev. 9, 629-636.

[11] L. Mei and P. Guan, 2017: Development of an atmospheric polarization Scheimpflug lidar system based on a time-division multiplexing scheme, Opt. Lett. 42, 3562-3565.

[12] L. Mei, P. Guan, and Z. Kong, 2017: Remote sensing of atmospheric $\mathrm{NO}_{2}$ by employing the continuous-wave differential absorption lidar technique, Opt. Exp. 25, A953-A962.

[13] L. Mei, P. Guan, Y. Yang, and Z. Kong, 2017: Atmospheric extinction coefficient retrieval and validation for the single-band Mie-scattering Scheimpflug lidar technique, Opt. Exp. 25, A628A638. 